University of Nebraska - Lincoln

DigitalCommons@University of Nebraska - Lincoln

Mammalogy Papers: University of Nebraska

State Museum

Museum, University of Nebraska State

January 1979

Rabbits (Genus Sylvilagus) of Nicaragua

Terry L. Yates

Texas Tech University, Lubbock, Texas

Hugh H. Genoways

University of Nebraska-Lincoln, h.h.genoways@gmail.com

J. Knox Jones Jr.

Texas Tech University, Lubbock, Texas

Follow this and additional works at: https://digitalcommons.unl.edu/museummammalogy

Part of the Zoology Commons

Yates, Terry L.; Genoways, Hugh H.; and Knox Jones, J. Jr., "Rabbits (Genus Sylvilagus) of Nicaragua" (1979). Mammalogy Papers: University of Nebraska State Museum. 77.

https://digitalcommons.unl.edu/museummammalogy/77

This Article is brought to you for free and open access by the Museum, University of Nebraska State at DigitalCommons@University of Nebraska - Lincoln. It has been accepted for inclusion in Mammalogy Papers: University of Nebraska State Museum by an authorized administrator of DigitalCommons@University of Nebraska Lincoln. 


\title{
Rabbits (Genus Sylvilagus) of Nicaragua
}

\author{
by \\ Terry L. YATES, Hugh H. GENOWAYS, \\ and J. Knox JONES, Jr.
}

Ce travail constitue une révision systématique des lapins (Sylvilagus) du Nicaragua. On a pu apprécier l'importance de la variation géographique d'après l'examen de 124 spécimens de Musée constituant 28 échantillons de $S$. floridanus du Honduras, du Nicaragua et de Costa-Rica, et 15 échantillons de $S$. brasiliensis du Nicaragua et de Costa-Rica.

Les particularités morphologiques de la peau et du crâne ont été soumises à des analyses statistiques. La variabilité géographique de $S$. floridanus permet de mettre en évidence que les représentants de l'espèce au Nicaragua sont plutôt référables à la forme $S$. $f$. cosiaricensis qu'à $S . f$. hondurensis comme on le croyait jusqu'alors. D'après ce que l'on sait de la variabilité géographique de $S$. brasiliensis, il ne paraît pas exister d'autre sous-espèce au Nicaragua que $S$. b. gabbi.

The genus Sylvilagus is represented in Nicaragua by two species, Sylvilagus brasiliensis and Sylvilagus floridanus. Nelson (1909) revised the genus and subsequent authors (Goldman, 1932 ; Harris, 1933; Hershkovitz, 1950) have treated certain Latin American populations taxonomically. Nevertheless, little has been published on Nicaraguan material because few specimens heretofore have been available for study from that country. Acquisition in recent years of a comparatively large series of rabbits from Nicaragua, together with extant specimens from Honduras and Costa Rica, has made possible a meaningful review of the genus Sylvilagus in this important Middle American region.

Our study was based on examination of 124 museum specimens, constituting 28 samples of Sylvilagus floridanus from Honduras, Nicaragua, and Costa Rica, and 15 samples of $S$. brasiliensis from Nicaragua and Costa Rica. The following 12 external and cranial measurements were recorded for each specimen used in analyses : total length ; length of tail ; length of hind foot ; length of ear (from notch) ; greatest length of skull ; zygomatic breadth ; interorbital breadth ; breadth of braincase ; length of palatal bridge ; length of maxillary toothrow; breadth of mesopterygoid fossa (least transverse diameter of the mesopterygoid fossa, measured on the ventral rim of the fossa on the outside of palatine bones posterior to the molar toothrow) ; length of nasals. All cranial measurements were taken to the nearest 0.1 millimeter with the aid of dial calipers. External measurements were taken directly from museum specimen labels as were measurements of testes and embryo lengths, and all are recorded in millimeters.

Individual, age, and secondary sexual variation were analysed with the aid of the univariate statistical system (UNIVAR) described by Power (1970). All specimens were assigned to one of three age classes. Means were calculated for each character and a single classification analysis of variance was used to

Mammalia, t. 43, n० 1, 1979. 
test for differences between age classes and sexes. Coefficients of variation (CV) were calculated to determine the extent of variability for each character.

Geographic variation was analyzed using both univariate (mean, standard deviation, and standard error) and multivariate statistics. To assess the degree of divergence among samples, a multivariate analysis of variance (MANOVA) and canonical analysis program from the statistical analysis system (SAS) designed and implemented by Barr and Goodnight (Service, 1972) were used. Canonical analysis of data aimed at providing weighted combinations of measurements that maximize the distinction between groups. This program extracts characteristic roots and vectors and computes mean canonical variates for each sample. The relative importance of each original variable to a particular

TABLE 1..- Results of analyses of variances among age classes of Sylvilagus brasiliensis from Río Mico, El Recreo, Zelaya, Nicaragua.

\begin{tabular}{|c|c|c|c|c|c|c|c|c|}
\hline Measurement & $\begin{array}{l}\text { Age } \\
\text { class }\end{array}$ & $N$ & $F$ & $\bar{x}$ & SD & $\mathrm{SE}$ & $\mathrm{CV}$ & Range \\
\hline \multirow[t]{2}{*}{ Total length } & 1 & 6 & \multirow{2}{*}{24.0} & 305.8 & 15.0 & 6.1 & 4.9 & $285.0-325.0$ \\
\hline & 2 & 4 & & 350.0 & 12.1 & 6.0 & 3.5 & $340.0-367.0$ \\
\hline \multirow[t]{2}{*}{ Length of tail } & $\begin{array}{l}3 \\
1\end{array}$ & $\begin{array}{l}6 \\
6\end{array}$ & \multirow{2}{*}{0.5} & $\begin{array}{r}346.0 \\
21.0\end{array}$ & $\begin{array}{r}30.7 \\
6.4\end{array}$ & $\begin{array}{r}12.5 \\
2.6\end{array}$ & $\begin{array}{r}8.9 \\
30.7\end{array}$ & $\begin{array}{r}309.0-385.0 \\
12.0-28.0\end{array}$ \\
\hline & 2 & 4 & & 18.5 & 2.6 & 1.3 & 14.8 & $15.0-21.0$ \\
\hline \multirow[t]{2}{*}{ Length of hind foot } & $\begin{array}{l}3 \\
1\end{array}$ & $\begin{array}{l}6 \\
6\end{array}$ & \multirow{2}{*}{5.1} & $\begin{array}{l}19.5 \\
69.5\end{array}$ & $\begin{array}{l}4.9 \\
4.4\end{array}$ & $\begin{array}{l}2.0 \\
1.8\end{array}$ & $\begin{array}{r}25.3 \\
6.3\end{array}$ & $\begin{array}{l}15.0-27.0 \\
63.0-75.0\end{array}$ \\
\hline & 2 & 4 & & 76.2 & 4.9 & 2.5 & 6.5 & $69.0-80.0$ \\
\hline \multirow[t]{2}{*}{ Length of ear } & $\begin{array}{l}3 \\
1\end{array}$ & $\begin{array}{l}6 \\
6\end{array}$ & \multirow{2}{*}{10.8} & $\begin{array}{l}75.7 \\
43.5\end{array}$ & $\begin{array}{l}1.9 \\
2.8\end{array}$ & $\begin{array}{l}0.8 \\
1.1\end{array}$ & $\begin{array}{l}2.5 \\
6.5\end{array}$ & $\begin{array}{l}73.0-78.0 \\
38.0-45.0\end{array}$ \\
\hline & 2 & 4 & & 48.2 & 0.5 & 0.3 & 1.0 & $48.0-49.0$ \\
\hline \multirow{2}{*}{$\begin{array}{l}\text { Greatest length of } \\
\text { skull }\end{array}$} & $\begin{array}{l}3 \\
1\end{array}$ & $\begin{array}{l}6 \\
6\end{array}$ & \multirow{2}{*}{14.6} & $\begin{array}{l}46.3 \\
59.6\end{array}$ & 2.1 & $\begin{array}{l}0.8 \\
2.0\end{array}$ & $\begin{array}{l}4.5 \\
5.9\end{array}$ & $\begin{array}{l}43.0-49.0 \\
55.6-62.3\end{array}$ \\
\hline & 2 & 4 & & 66.7 & 1.3 & 0.6 & 1.9 & $65.4-68.0$ \\
\hline \multirow[t]{2}{*}{ Zygomatic breadth } & $\begin{array}{l}3 \\
1\end{array}$ & $\begin{array}{l}6 \\
6\end{array}$ & \multirow{2}{*}{15.5} & $\begin{array}{l}67.6 \\
29.6\end{array}$ & $\begin{array}{l}4.6 \\
1.0\end{array}$ & $\begin{array}{l}1.9 \\
0.4\end{array}$ & $\begin{array}{l}6.8 \\
3.4\end{array}$ & $\begin{array}{l}62.2-74.0 \\
28.2-30.8\end{array}$ \\
\hline & 2 & 4 & & 31.8 & 0.6 & 0.3 & 1.9 & 31.3- 32.5 \\
\hline \multirow[t]{2}{*}{ Interorbital breadth } & $\begin{array}{l}3 \\
1\end{array}$ & $\begin{array}{l}6 \\
6\end{array}$ & 8.3 & $\begin{array}{l}31.8 \\
12.9\end{array}$ & $\begin{array}{l}1.3 \\
0.7\end{array}$ & $\begin{array}{l}0.5 \\
0.3\end{array}$ & $\begin{array}{l}4.1 \\
3.6\end{array}$ & $\begin{array}{l}30.2-33.2 \\
11.9-14.0\end{array}$ \\
\hline & 2 & 4 & 0.3 & 14.0 & 0.4 & 0.2 & 2.5 & 13.7- 14.4 \\
\hline \multirow[t]{2}{*}{ Breadth of braincase } & $\begin{array}{l}3 \\
1\end{array}$ & $\begin{array}{l}6 \\
6\end{array}$ & 4.0 & $\begin{array}{l}14.5 \\
22.0\end{array}$ & $\begin{array}{l}1.4 \\
0.7\end{array}$ & $\begin{array}{l}0.6 \\
0.3\end{array}$ & $\begin{array}{l}9.9 \\
3.1\end{array}$ & $\begin{array}{l}12.8-16.1 \\
21.1-\frac{1}{22.8}\end{array}$ \\
\hline & 2 & 4 & \multirow{2}{*}{0.2} & 22.8 & 0.5 & 0.2 & 2.1 & $22.3-23.3$ \\
\hline \multirow{2}{*}{$\begin{array}{l}\text { Length of palatal } \\
\text { bridge }\end{array}$} & $\begin{array}{l}3 \\
1\end{array}$ & $\begin{array}{l}6 \\
6\end{array}$ & & $\begin{array}{r}22.6 \\
6.6\end{array}$ & $\begin{array}{l}0.7 \\
0.3\end{array}$ & $\begin{array}{l}0.3 \\
0.1\end{array}$ & $\begin{array}{l}3.3 \\
4.9\end{array}$ & $\begin{array}{r}21.9-23.8 \\
6.1-6.9\end{array}$ \\
\hline & 2 & 4 & 4.2 & 7.0 & 0.3 & 0.2 & 4.6 & $6.8-7.5$ \\
\hline \multirow{2}{*}{$\begin{array}{l}\text { Length of maxillary } \\
\text { toothrow }\end{array}$} & $\begin{array}{l}3 \\
1\end{array}$ & $\begin{array}{l}6 \\
6\end{array}$ & \multirow{2}{*}{15.2} & $\begin{array}{r}6.7 \\
11.8\end{array}$ & $\begin{array}{l}0.4 \\
0.5\end{array}$ & $\begin{array}{l}0.2 \\
0.2\end{array}$ & $\begin{array}{l}5.5 \\
4.1\end{array}$ & $\begin{array}{r}6.4 \\
11.1-12.3\end{array}$ \\
\hline & 2 & 4 & & 12.8 & 0.2 & 0.1 & 1.6 & $12.5-12.9$ \\
\hline \multirow{2}{*}{$\begin{array}{l}\text { Breadth of meso- } \\
\text { pterygoid fossa }\end{array}$} & $\begin{array}{l}3 \\
1\end{array}$ & $\begin{array}{l}6 \\
6\end{array}$ & \multirow{2}{*}{4.9} & $\begin{array}{r}12.8 \\
8.3\end{array}$ & $\begin{array}{l}1.1 \\
0.6\end{array}$ & $\begin{array}{l}0.5 \\
0.2\end{array}$ & $\begin{array}{l}8.9 \\
6.9\end{array}$ & $\begin{array}{r}11.4-13.9 \\
7.7-9.3\end{array}$ \\
\hline & 2 & 4 & & 9.0 & 0.4 & 0.2 & 4.1 & $8.6-9.5$ \\
\hline \multirow[t]{3}{*}{ Length of nasal } & $\begin{array}{l}3 \\
1\end{array}$ & $\begin{array}{l}6 \\
6\end{array}$ & 0.0 & $\begin{array}{r}9.0 \\
25.1\end{array}$ & $\begin{array}{l}0.5 \\
1.6\end{array}$ & $\begin{array}{l}0.2 \\
0.7\end{array}$ & $\begin{array}{l}5.8 \\
6.5\end{array}$ & $\begin{array}{r}8.4-9.6 \\
22.3-26.3\end{array}$ \\
\hline & 2 & 4 & \multirow{2}{*}{$\begin{array}{r}10.5 \\
0.2\end{array}$} & 28.0 & 0.8 & 0.4 & 2.7 & $26.9 \cdot 28.6$ \\
\hline & 3 & 6 & & 28.6 & 2.5 & 1.0 & 8.9 & $25.8-32.6$ \\
\hline
\end{tabular}


canonical variate was computed by multiplying the vector variable coefficient by the median value of the dependent variable, summing all variable values for a particular vector, and then computing the percent of relative importance of each variable per vector.

Field work in Nicaragua was supported by a contract (DA-49-193-MD-2215) from the U. S. Army Research and Development Command. Some of the laboratory work was supported by a grant (GB-28957) from the National Science Foundation.

We are grateful to officials of the following institutions for loan of specimens : American Museum of Natural History (AMNH) ; Museum of Natural History, University of Kansas (KU); Museum of Zoology University of Michigan (UMMZ) ; National Museum of Natural History (USNM).

\section{SYLVILAGUS BRASILIENSIS}

\section{NONGEOGRAPHIG VARIATION}

Three types of nongeographic variation - variation with age, secondary sexual variation, and individual variation - have been treated. An understanding of these kinds of variation is necessary in order to properly interpret geographic variation in both species of Nicaraguan Sylvilagus.

Variation with age. - All specimens were originally assigned to one of three age classes, which were defined as follows : class I, suture between basisphenoid and basioccipital bones not closed; class II, suture between basisphenoid and basioccipital bones closed but clearly visible to the naked eye as a thin line ; class III, suture between basisphenoid and basioccipital bones completely ossified and not visible to the naked eye.

Analysis of variance was used to test each of 12 external and cranial measurements for significant differences between age classes in a sample from the south side of the Rfo Mico, El Recreo, Zelaya, Nicaragua (Table 1). Signiflcant differences $(P<.05)$ between age classes I and II were noted for all measurements except length of tail. No significant differences were found between age classes II and III. Therefore, animals in class I were excluded from further analysis and those of classes II and III were combined for study of geographic variation.

Secondary sexual variation. - Analysis of variance was used to test each of 12 external and cranial characters for significant differences between males and females in a grouped sample from the Nicaraguan departments of Zelaya, Jinotega, and Matagalpa (Table 2). No significant sexual differences were found in any character. Therefore, the sexes were treated together in all subsequent analyses.

Individual variation. - As expected, cranial measurements were found to be less variable than external measurements (Table 2). This is due, in part at least, to the greater difficulty in accurately taking external measurements. 
TABLE 2. - Resuits of analysis of variance between males and females for a grouped sample of Sylvilagus brasiliensis from the departments of Zelaya, Jinotega, and Matagalpa, Nicaragua, and a sample of Sylvilegus floridanus from the vicinity of Rivas, Rivas, Nicaragua.

\begin{tabular}{|c|c|c|c|c|c|c|c|c|}
\hline Measurement & Sex & $N$ & $F$ & $\bar{x}$ & SD & $\mathrm{SE}$ & CV & Range \\
\hline \multicolumn{9}{|c|}{ Sylvilagus brasiliensis } \\
\hline \multirow[t]{2}{*}{ Total length } & Malc & 8 & \multirow{2}{*}{0.04} & 351.38 & 10.47 & 3.70 & 2.98 & $340.0-367.0$ \\
\hline & Female & 6 & & 354.00 & 34.44 & 14.06 & 9.73 & $309.0-395.0$ \\
\hline \multirow[t]{2}{*}{ Length of tail } & Male & 8 & \multirow{2}{*}{0.97} & 19.00 & 4.00 & 1.41 & 21.05 & $15.0-27.0$ \\
\hline & Female & 6 & & 17.00 & 3.41 & 1.39 & 20.03 & $13.0-22.0$ \\
\hline \multirow[t]{2}{*}{ Length of hind foot } & Male & 8 & \multirow{2}{*}{1.75} & 75.00 & 4.11 & 1.45 & 5.47 & $69.0-80.0$ \\
\hline & Fermale & 6 & & 78.00 & 4.34 & 1.77 & 5.56 & $72.0-85.0$ \\
\hline \multirow[t]{2}{*}{ Length of ear } & MaIe & 8 & \multirow{2}{*}{0.40} & 47.25 & 2.12 & 0.75 & 1.75 & $43.0-49.0$ \\
\hline & Female & 6 & & 46.67 & 0.82 & 0.33 & 4.49 & $46.0-48.0$ \\
\hline \multirow{2}{*}{$\begin{array}{l}\text { Greatest length of } \\
\text { skult }\end{array}$} & Male & 9 & \multirow{2}{*}{0.00} & 68.39 & 2.51 & 0.84 & 3.68 & $65.4-74.0$ \\
\hline & Female & 6 & & 68.47 & 4.34 & 1.77 & 6.34 & $62.2 \sim 74.2$ \\
\hline \multirow[t]{2}{*}{ Zygomatic breadth } & Male & 9 & \multirow{2}{*}{0.20} & 32.11 & 0.54 & 0.18 & 1.67 & $31.3-32.7$ \\
\hline & Female & 6 & & 32.32 & 1.25 & 0.51 & 3.86 & $30.2-33.6$ \\
\hline \multirow[t]{2}{*}{ Interorbital breadth } & Male & 9 & \multirow{2}{*}{0.18} & 14.63 & 0.79 & 0.26 & 5.40 & $13.7-16.1$ \\
\hline & Fetnale & 6 & & 14.40 & 1.38 & 0.56 & 9.56 & $12.8-16.3$ \\
\hline \multirow[t]{2}{*}{ Breadth of braincase } & Male & 9 & \multirow{2}{*}{0.07} & 23.02 & 0.70 & 0.24 & 3.06 & $22.3=24.4$ \\
\hline & Female & 6 & & 22.92 & 0.97 & 0.40 & 4.25 & $21.9 \cdot 24.4$ \\
\hline \multirow{2}{*}{$\begin{array}{l}\text { Length of palutal } \\
\text { bridge }\end{array}$} & Mate & 9 & \multirow{2}{*}{1.06} & 6.98 & 0.33 & 0.11 & 4.69 & $6.5-7.5$ \\
\hline & Temale & 6 & & 6.78 & 0.40 & 0.16 & 5.93 & $6.3 \cdot 7.2$ \\
\hline $\begin{array}{l}\text { Length of maxillary } \\
\text { toothrow }\end{array}$ & Male & 9 & & 13.21 & 0.52 & 0.17 & 3.92 & $12.5+13.9$ \\
\hline & Female & 6 & & 12.92 & 0.85 & 0.35 & 6.61 & $11.4 \cdot 14.0$ \\
\hline $\begin{array}{l}\text { Breadth of meso- } \\
\text { pterygoid fossa }\end{array}$ & Male & 9 & & 9.30 & 0.43 & 0.14 & 4.66 & 8.4- 9.7 \\
\hline & Female & 6 & & 9.12 & 0.61 & 0.25 & 6.70 & $8.6-10.1$ \\
\hline Length of nasals & Malc & 9 & & 28.86 & 1.57 & 0.52 & 5.44 & $26.9-32.6$ \\
\hline & Female & 6 & 1.0 & 28.92 & 2.04 & 0.83 & 7.04 & $25.8 \cdot 31.6$ \\
\hline & & & Sylvil & ภำ & & & & \\
\hline Total length & Male & 10 & & 439.2 & 46.2 & 14.6 & 10.5 & $400.0-564.0$ \\
\hline & Female & 12 & & 446.8 & 42.0 & 12.1 & 9.4 & $380.0-563.0$ \\
\hline Length of tail & Male & 10 & & 43.2 & 7.1 & 2.3 & 16.5 & $30.0-58.0$ \\
\hline & Female & 12 & & 48.7 & 7.6 & 2.2 & 15.6 & $30.0 \cdot 56.0$ \\
\hline Length of hind foot & Male & 10 & & 82.8 & 16.2 & 5.1 & 19.5 & $50.0-94.0$ \\
\hline & Femak & 12 & & 78.0 & 22.6 & 6.5 & 29.0 & $40.0-100.0$ \\
\hline Length of ear & Male & 10 & & 58.5 & 6.1 & 1.9 & 10.5 & $50.0 \cdot 70.0$ \\
\hline & Fernale & 12 & 0.00 & 59.8 & 4.9 & 1.4 & 8.1 & $52.0-79.0$ \\
\hline Greatest length of & Male & 9 & & 77.8 & 1.6 & 0.5 & 2.0 & $76.4-80.9$ \\
\hline & Female & 11 & & 77.9 & 2.4 & 0.7 & 3.0 & $74.7 \cdot 81.9$ \\
\hline Zygomatic breadth & Male & 7 & & 34.8 & 0.6 & 0.2 & 1.6 & $24.0-35.7$ \\
\hline & Female & 10 & & 35.3 & 0.8 & 0.3 & 2.3 & $33.5-36.5$ \\
\hline Interorbital breadth & Male & 9 & & 18.9 & 0.7 & 0.2 & 3.6 & 17.9- 20.1 \\
\hline & Female & 11 & & 19.2 & 1.0 & 0.3 & 5.0 & $17.6 \cdot 20.7$ \\
\hline Breadth of braincase & Male & 8 & & 25.6 & 0.4 & 0.1 & 1.4 & $25.0-26.1$ \\
\hline & & 10 & & 26.1 & 08 & 0.3 & 3.0 & $24.8-27.2$ \\
\hline Length of palatal & Male & 9 & & 7.6 & 0.4 & 0.1 & 5.3 & $6.9-8.4$ \\
\hline & Femali & 11 & & 7.4 & 0.5 & 0.1 & 6.8 & $6.5-8.2$ \\
\hline Length of inaxillary & Male & 9 & & 14.0 & 0.2 & 0.1 & 1.7 & $13.7-14.3$ \\
\hline & Female & 11 & & 13.8 & 05 & 0.1 & 3.7 & $13.0-14.7$ \\
\hline Breadth of meso- & Male & 8 & & 9.4 & 0.1 & 0.1 & 1.6 & $9.1-9.6$ \\
\hline & Fennale & 10 & & 9.3 & 0.3 & 0.1 & 3.5 & $8.9-10.0$ \\
\hline Length of nasals & & 9 & & 34.8 & 1.3 & 0.4 & 3.8 & $33.3-37.0$ \\
\hline & Female & 11 & & 35.0 & 1.1 & 0.3 & 3.0 & $33.6-37.2$ \\
\hline
\end{tabular}


In most characters, males tended to be less variable than did females. The average coefficient of variation for males, considering all characters, was 5.31, whereas for females it was 7.50. As expected, the degree of difference was less when only cranial measurements were considered, an average of 4.06 for males and 6.29 for females.

Considering cranial measurements, zygomatic breadth and breadth of the braincase were found to be the least variable. With the exception of interorbital breadth, all cranial measurements exhibited coefficients of variation of 7.04 or less. Those for external measurements ranged from a low of 1.75 for length of ear to a high of 21.05 for length of tail.

Because no quantitative measure of pelage color was used, no attempt was made to analyze statistically individual color variation. Nelson (1909) found pelage coloration in cottontails to be highly variable.

\section{Geographic Variation}

Analysis of geographic variation was based on the examination of 26 specimens of Sylvilagus brasiliensis from eight localities in Nicaragua and Costa Rica. Most specimens examined were pooled geographically into four grouped localities as follows (localities taken with reference to the nearest town) : area 1-Jinotega (Hacienda la Trampa) and Matagalpa (Muy Muy, San Ramón, Matagalpa, Hacienda Tepeyac, Tuma), Nicaragua ; area 2-Zelaya (El Recreo), Nicaragua ; area 3-Alajuela (Vijagual), Costa Rica ; area 4Cartago (Santa Teresa), Costa Rica. The samples from Costa Rica contained only one specimen each. Standard statistics (mean, standard deviation, and standard error) were calculated for all external and cranial measurements used in the study (Table 3 ). Because there were no significant differences between samples from areas 1 and 2 , no multivariate analyses were conducted.

In general, specimens from area 1 (the more northerly departments of Jinotega and Matagalpa) averaged slightly larger than did specimens from Zelaya (area 2). With the exception of length of tail and length of ear, all external and cranial measurements averaged slightly larger for specimens from area 1 than for those from area 2 . Both specimens examined from Costa Rica (areas 3 and 4) are larger than those from Nicaragua. Based on our assessment of geographic variation in $S$. brasiliensis, there appears to be only one subspecies in Nicaragua.

\section{Sylvilagus brasiliensis gabbi (J. A. Allen)}

1877. - Lepus brasiliensis var. gabbi J. A. Allen, Bull. U. S. Geol. Surv. Territories, 11 : 349, August. Type locality, Costa Rica and Chiriqui ; restricted by Nelson (N. Amer. Fauna, $29: 259$, August 31, 1909), by designation of type specimen, to Talamanca ( $=\mathrm{Si}-$ purio, Río Sixaola, near Caribbean Coast), Costa Rica.

1950. - Sylvilagus brasiliensis gabbi, Hershkovitz, Proc. U. S. Nat. Mus., $100: 351$, May 26.

Distribution. - Eastern Honduras, Nicaragua, Costa Rica, and western Panamá. 


\begin{tabular}{|c|c|c|c|c|c|c|c|c|c|c|c|c|c|}
\hline Sample & Statistics & $\begin{array}{l}\text { Total } \\
\text { length }\end{array}$ & $\begin{array}{c}\text { Longth } \\
\text { of } \\
\text { tail } \\
\end{array}$ & $\begin{array}{l}\text { Length } \\
\text { of } \\
\text { hind } \\
\text { foot } \\
\end{array}$ & Ear & $\begin{array}{c}\text { Greatest } \\
\text { length } \\
\text { of } \\
\text { skull }\end{array}$ & $\begin{array}{c}\text { Zygo- } \\
\text { matic } \\
\text { breadth }\end{array}$ & $\begin{array}{c}\text { Inter- } \\
\text { orbital } \\
\text { bread th }\end{array}$ & $\begin{array}{l}\text { Bread th } \\
\text { of } \\
\text { braincuse }\end{array}$ & $\begin{array}{c}\text { Length } \\
\text { of } \\
\text { palatal } \\
\text { bridge }\end{array}$ & $\begin{array}{l}\text { Length of } \\
\text { maxillary } \\
\text { toothrow }\end{array}$ & $\begin{array}{l}\text { Breadth } \\
\text { of Mesop- } \\
\text { terygoid } \\
\text { fossa }\end{array}$ & $\begin{array}{c}\text { Length } \\
\text { of } \\
\text { nasile }\end{array}$ \\
\hline \multicolumn{14}{|c|}{ Sylvilagus braslijensis } \\
\hline$I$ & $\begin{array}{l}\text { Mean } \\
N \\
\text { SD } \\
\text { SE } \\
\text { Minimum } \\
\text { Maximum }\end{array}$ & $\begin{array}{c}354.0 \\
5 \\
28.0 \\
12.5 \\
320 \\
395\end{array}$ & $\begin{array}{l}17.0 \\
5 \\
3.1 \\
1.4 \\
13 \\
20\end{array}$ & $\begin{array}{l}77.0 \\
5 \\
6.1 \\
2.7 \\
70 \\
85\end{array}$ & $\begin{array}{c}46.6 \\
5 \\
1.1 \\
0.5 \\
45 \\
48\end{array}$ & $\begin{array}{c}69.4 \\
6 \\
3.2 \\
1.3 \\
64.0 \\
74.2\end{array}$ & $\begin{array}{c}32.5 \\
6 \\
0.7 \\
0.3 \\
31.0 \\
33.6\end{array}$ & $\begin{array}{c}14.7 \\
6 \\
1.1 \\
0.4 \\
13.0 \\
16.3\end{array}$ & $\begin{array}{c}23.3 \\
6 \\
1.0 \\
0.4 \\
22.0 \\
24.4\end{array}$ & $\begin{array}{l}6.9 \\
6 \\
0.4 \\
0.2 \\
6.3 \\
7.2\end{array}$ & $\begin{array}{c}13.3 \\
6 \\
0.5 \\
0.2 \\
12.3 \\
14.0\end{array}$ & $\begin{array}{l}9.8 \\
6 \\
0.6 \\
0.2 \\
8.4 \\
10.1\end{array}$ & $\begin{array}{c}29.2 \\
6 \\
1.5 \\
0.6 \\
27.5 \\
31.6\end{array}$ \\
\hline II & $\begin{array}{l}\text { Mean } \\
N \\
\text { SD } \\
\text { SE } \\
\text { Minimum } \\
\text { Maximum }\end{array}$ & $\begin{array}{c}351.0 \\
9 \\
21.1 \\
7.0 \\
309 \\
385\end{array}$ & $\begin{array}{c}18.8 \\
9 \\
4.1 \\
1.4 \\
15 \\
27\end{array}$ & $\begin{array}{l}75.9 \\
9 \\
3.4 \\
1.1 \\
69 \\
80\end{array}$ & $\begin{array}{c}47.2 \\
9 \\
1.9 \\
0.6 \\
43 \\
49\end{array}$ & $\begin{array}{c}67.8 \\
9 \\
3.3 \\
1.1 \\
62.2 \\
74.0\end{array}$ & $\begin{array}{c}32.0 \\
9 \\
0.9 \\
0.3 \\
30.2 \\
33.2\end{array}$ & $\begin{array}{c}14.5 \\
9 \\
1.0 \\
0.3 \\
12.8 \\
16.1\end{array}$ & $\begin{array}{c}22.8 \\
9 \\
0.6 \\
0.2 \\
21.9 \\
23.8\end{array}$ & $\begin{array}{l}6.9 \\
, 9 \\
0.4 \\
0.1 \\
6.4 \\
7.5\end{array}$ & $\begin{array}{c}13.0 \\
9 \\
0.7 \\
0.2 \\
11.4 \\
13.9\end{array}$ & $\begin{array}{l}9.1 \\
9 \\
0.4 \\
0.1 \\
8.4 \\
9.6\end{array}$ & $\begin{array}{c}28.6 \\
9 \\
1.9 \\
0.6 \\
25.8 \\
32.6\end{array}$ \\
\hline III & $\begin{array}{l}\text { AMNH } \\
141913\end{array}$ & 380 & 20 & 80 & 45 & 72.4 & 33.5 & 16.8 & 23.8 & 7.4 & 13.8 & 9.5 & 30.0 \\
\hline IV & $\begin{array}{l}\text { AHNH } \\
141869\end{array}$ & 365 & 25 & 80 & 45 & 68.9 & 34.1 & 15.0 & 22.7 & 7.3 & 14.0 & 10.0 & 26.3 \\
\hline \multicolumn{14}{|c|}{ Sylvilogus floridanus } \\
\hline I & $\begin{array}{l}\text { Mean } \\
N \\
\text { SD } \\
\text { SE } \\
\text { Minimum } \\
\text { Maximum }\end{array}$ & $\begin{array}{c}424.4 \\
8 \\
17.6 \\
6.2 \\
406 \\
450\end{array}$ & $\begin{array}{c}34.6 \\
8 \\
5.1 \\
1.8 \\
26 \\
40\end{array}$ & $\begin{array}{c}84.6 \\
7 \\
3.0 \\
1.2 \\
80 \\
90\end{array}$ & $\begin{array}{c}59.7 \\
8 \\
1.9 \\
0.7 \\
58 \\
63\end{array}$ & $\begin{array}{r}75.3 \\
8 \\
2.2 \\
0.8 \\
70.7 \\
77.2\end{array}$ & $\begin{array}{c}35.8 \\
7 \\
0.8 \\
0.3 \\
34.8 \\
36.8\end{array}$ & $\begin{array}{r}19.0 \\
8 \\
0.9 \\
0.3 \\
17.1 \\
19.7\end{array}$ & $\begin{array}{c}25.7 \\
8 \\
0.7 \\
0.2 \\
24.6 \\
26.5\end{array}$ & $\begin{array}{l}7.6 \\
8 \\
0.4 \\
0.2 \\
6.9 \\
8.1\end{array}$ & $\begin{array}{c}14.0 \\
8 \\
0.4 \\
0.1 \\
13.6 \\
14.8\end{array}$ & $\begin{array}{l}9.1 \\
7 \\
0.3 \\
0.1 \\
8.7 \\
9.4\end{array}$ & $\begin{array}{r}32.7 \\
8 \\
1.8 \\
0.6 \\
30.0 \\
35.0\end{array}$ \\
\hline II & $\begin{array}{l}\text { Mean } \\
N \\
\text { SD } \\
\text { SE } \\
\text { Minimum } \\
\text { Maximum }\end{array}$ & $\begin{array}{c}439.4 \\
5 \\
57.7 \\
25.8 \\
401 \\
540\end{array}$ & $\begin{array}{l}41.0 \\
5 \\
9.6 \\
4.3 \\
32 \\
55\end{array}$ & $\begin{array}{l}93.4 \\
5 \\
2.9 \\
1.3 \\
89 \\
96\end{array}$ & $\begin{array}{l}57.4 \\
5 \\
3.4 \\
1.5 \\
53 \\
61\end{array}$ & $\begin{array}{c}74.8 \\
5 \\
1.6 \\
0.7 \\
72.6 \\
76.8\end{array}$ & $\begin{array}{c}34.1 \\
5 \\
0.7 \\
0.3 \\
33.3 \\
35.0\end{array}$ & $\begin{array}{c}18.7 \\
5 \\
0.9 \\
0.4 \\
17.6 \\
19.7\end{array}$ & $\begin{array}{c}25.4 \\
5 \\
0.4 \\
0.2 \\
24.8 \\
25.9\end{array}$ & $\begin{array}{l}7.4 \\
5 \\
0.4 \\
0.2 \\
6.8 \\
8.0\end{array}$ & $\begin{array}{c}14.0 \\
5 \\
0.5 \\
0.2 \\
13.3 \\
14.4\end{array}$ & $\begin{array}{l}8.7 \\
4 \\
0.2 \\
0.1 \\
8.5 \\
9.0\end{array}$ & $\begin{array}{r}32.7 \\
5 \\
1.2 \\
0.5 \\
31.4 \\
34.4\end{array}$ \\
\hline III & $\begin{array}{l}\text { Mean } \\
\text { SD } \\
\text { SE } \\
\text { Minimum } \\
\text { Maximum }\end{array}$ & $\begin{array}{c}430.5 \\
4.5 \\
22.2 \\
11.2 \\
298 \\
449\end{array}$ & $\begin{array}{c}40.5 \\
4 \\
10.0 \\
5.0 \\
32 \\
55\end{array}$ & $\begin{array}{c}94.2 \\
4 \\
3.9 \\
1.9 \\
89 \\
98\end{array}$ & $\begin{array}{l}61.5 \\
4.6 \\
2.6 \\
1.3 \\
59 \\
65\end{array}$ & $\begin{array}{c}77.6 \\
4 \\
2.4 \\
1.2 \\
74.6 \\
80.0\end{array}$ & $\begin{array}{r}35.4 \\
4.3 \\
1.3 \\
0.6 \\
33.6 \\
36.5\end{array}$ & $\begin{array}{r}19.7 \\
4 \\
0.8 \\
0.4 \\
18.6 \\
20.3\end{array}$ & $\begin{array}{c}26.5 \\
4 \\
0.9 \\
0.4 \\
25.2 \\
27.1\end{array}$ & $\begin{array}{l}7.5 \\
4 \\
0.1 \\
0.1 \\
7.3 \\
7.6\end{array}$ & $\begin{array}{r}14.2 \\
4.2 \\
0.3 \\
0.2 \\
13.8 \\
14.6\end{array}$ & $\begin{array}{l}9.0 \\
4 \\
0.5 \\
0.3 \\
8.3 \\
9.4\end{array}$ & $\begin{array}{r}26.0 \\
4.4 \\
1.4 \\
0.7 \\
34.2 \\
37.3\end{array}$ \\
\hline IV & $\begin{array}{l}\text { Mean } \\
N \\
\text { SD } \\
\text { SE } \\
\text { Minimum } \\
\text { Maximum }\end{array}$ & $\begin{array}{c}450.0 \\
3 \\
65.2 \\
37.6 \\
407 \\
525\end{array}$ & $\begin{array}{l}37.3 \\
3 \\
7.5 \\
4.3 \\
30 \\
45\end{array}$ & $\begin{array}{c}89.3 \\
3 \\
1.5 \\
0.9 \\
88 \\
91\end{array}$ & $\begin{array}{l}61.7 \\
3 \\
2.1 \\
1.2 \\
60 \\
64\end{array}$ & $\begin{array}{c}75.1 \\
4 \\
1.9 \\
0.9 \\
72.8 \\
76.8\end{array}$ & $\begin{array}{c}34.7 \\
4 \\
0.9 \\
0.4 \\
34.0 \\
36.0\end{array}$ & $\begin{array}{r}19.6 \\
4 \\
1.7 \\
0.6 \\
18.6 \\
21.2\end{array}$ & $\begin{array}{c}25.8 \\
4 \\
1.2 \\
0.6 \\
24.5 \\
27.5\end{array}$ & $\begin{array}{l}6.9 \\
4.9 \\
0.9 \\
0.4 \\
5.8 \\
7.8\end{array}$ & $\begin{array}{c}14.1 \\
4 \\
0.4 \\
0.2 \\
13.7 \\
14.6\end{array}$ & $\begin{array}{l}9.2 \\
4 \\
0.2 \\
0.1 \\
9.0 \\
9.5\end{array}$ & $\begin{array}{r}34.3 \\
4.0 \\
1.0 \\
0.5 \\
33.4 \\
35.4\end{array}$ \\
\hline $\mathbf{V}$ & $\begin{array}{l}\text { Mean } \\
N \\
\text { SD } \\
\text { SE } \\
\text { Minimum } \\
\text { Maximum }\end{array}$ & $\begin{array}{l}475.6 \\
15 \\
61.2 \\
23.1 \\
415 \\
564\end{array}$ & $\begin{array}{c}50.8 \\
15 \\
5.1 \\
1.9 \\
44 \\
58\end{array}$ & $\begin{array}{c}93.1 \\
15 \\
2.2 \\
0.8 \\
90 \\
97\end{array}$ & $\begin{array}{l}62.0 \\
15 \\
1.5 \\
0.6 \\
60 \\
64\end{array}$ & $\begin{array}{l}77.7 \\
15 \\
1.4 \\
0.5 \\
75.9 \\
80.0\end{array}$ & $\begin{array}{c}35.0 \\
15 \\
1.0 \\
0.4 \\
33.5 \\
36.5\end{array}$ & $\begin{array}{r}19.0 \\
15 \\
1.0 \\
0.4 \\
17.9 \\
20.5\end{array}$ & $\begin{array}{c}25.9 \\
15 \\
0.8 \\
0.3 \\
24.8 \\
27.2\end{array}$ & $\begin{array}{c}7.5 \\
15 \\
0.4 \\
0.1 \\
6.7 \\
7.8\end{array}$ & $\begin{array}{c}14.1 \\
15 \\
0.3 \\
0.1 \\
13.8 \\
14.7\end{array}$ & $\begin{array}{c}9.4 \\
15 \\
0.1 \\
0.1 \\
9.3 \\
9.6\end{array}$ & $\begin{array}{r}34.6 \\
15 \\
0.9 \\
0.4 \\
33.3 \\
35.9\end{array}$ \\
\hline $\mathbf{V I}$ & $\begin{array}{l}\text { KU } \\
115230\end{array}$ & 410 & 54 & 87 & 62 & 74.5 & 34.6 & 18.3 & 26.0 & 7.1 & 13.5 & 8.9 & 33.0 \\
\hline VI & $\begin{array}{l}\text { KU } \\
115229\end{array}$ & 455 & 50 & 91 & 61 & 76.6 & 34.3 & 19.0 & 25.5 & 6.9 & 13.9 & 9.1 & 34.7 \\
\hline vII & $\begin{array}{l}\text { Mean } \\
\mathbf{N} \\
\text { Minimum } \\
\text { Maximum }\end{array}$ & $\begin{array}{c}374.2 \\
4 \\
385 \\
417\end{array}$ & $\begin{array}{l}57.5 \\
2 \\
57 \\
58\end{array}$ & $\begin{array}{c}86.0 \\
4 \\
74 \\
97\end{array}$ & $\begin{array}{l}66.5 \\
2 \\
66 \\
67\end{array}$ & $\begin{array}{c}73.9 \\
4 \\
72.1 \\
75.0\end{array}$ & $\begin{array}{c}34.0 \\
4 \\
33.1 \\
34.5\end{array}$ & $\begin{array}{c}13.1 \\
4 \\
17.3 \\
18.9\end{array}$ & $\begin{array}{c}24.8 \\
4 \\
24.4 \\
25.3\end{array}$ & $\begin{array}{l}7.0 \\
4 \\
6.8 \\
7.1\end{array}$ & $\begin{array}{c}13.4 \\
4 \\
13.1 \\
14.0\end{array}$ & $\begin{array}{l}9.5 \\
4.5 \\
9.0 \\
9.8\end{array}$ & $\begin{array}{c}34.1 \\
4 \\
33.7 \\
34.9\end{array}$ \\
\hline $\mathbf{L X}$ & $\begin{array}{l}\text { KU } \\
115225\end{array}$ & 403 & 35 & 87 & 62 & 74.8 & 34.1 & 17.4 & 26.2 & 7.6 & 13.4 & 8.9 & 33.0 \\
\hline
\end{tabular}

Remarks. - Sylvilagus brasiliensis gabbi is restricted in Nicaragua to the eastern more humid half of the country (Fig. 3). It occurs commonly in the tropical rain forests of that region and is often observed along forest trails. $S$. brasiliensis has been taken sympatrically with the larger $S$. floridanus only at Ocotal and Jalapa in Nueva Segovia, but the two species occur in close association in the ecotonal areas of the north-central highlands of Nicaragua in the vicinity of Jinotega and Matagalpa. The two species are morphologically distinct, $S$. brasiliensis being the smaller of the two with a shorter tail that is dingy buff instead of white below. $S$. brasiliensis also tends to be much darker in color than floridanus.

Testes measurements for adult males from El Recreo are as follows (dates of capture in parentheses ) : 22 (16 June) ; 16 (19 June) ; 20, 27 (20 June) ; 30, 31 (21 June); 18, 23, 30 (23 June); 11 (25 July). A pregnant female was taken 
at $3 \mathrm{mi}$. E San Ramón on 11 May. This specimen carried four embryos that measured 16 in crown-rump length. A lactating female was taken on 19 June at El Recreo. Nonpregnant females were taken on 29 July, 30 July (two individuals), and 11 August.

Specimens examined. -- Total number 38 as follows (localities in italics are not plotted on Fig. 3) : Nicaragua. Jinotega : Hda. La Trampa, 2 (1 KU, 1 USNM ; Peña Blanca, 1 (AMNH). Matagalpa : Matagalpa, 2 (AMNH) ; $10 \mathrm{mi}$. NW Muy Muy, 1 (KU) ; $3 \mathrm{mi}$. E San Ramón, 1 (KU) ; Finca Tepayac, $101 / 2 \mathrm{~km}$. N, $9 \mathrm{~km}$. E Matagalpa, 4 (2 KU, 2 USNM) ; Tuma, 1 (AMNH) ; Vijagua, 1 (AMNH). Nueva Segovia : Jalapa, 1 (AMNH); Ocotal, 1 (AMNH). Zelaya : S side Río Mico, El Recreo, 18 (KU) ; El Recreo, 3 (USNM). - Costa Rica. Cartago : Sta. Teresa, 1 (AMNH) Alajuela : Vijagual 1 (AMNH).

\section{SYLVILAGUS FLORIDANUS}

\section{NongeographIG VARIATION}

Secondary sexual variation and individual variation were analyzed in a sample of Sylvilagus floridanus from Hacienda Amayo, Rivas, Nicaragua. Because of a lack of juvenile and subadult specimens, variation with age was not analyzed.

Secondary sexual variation. - Analysis of variance was used to test each of 12 external and cranial characters for significant differences between males and females (Table 2). Significant secondary sexual differences were found only in one character (length of tail). Males and females, therefore, were combined in subsequent analyses. The one character that varied with sex in our specimens, length of tail, was not included in other statistical analyses.

Individual variation. - Individual variation, as determined by coefficients of variation, was less for cranial measurements than for external measurements (Table 2). Coefficients of variation for external measurements averaged 14.25 for males and $\mathbf{1 5 . 5 2}$ for females. Because these values are undesirably high and because length of tail was found to be significantly different between sexes, all external measurements were eliminated from the multivariate portion of the study. Coefficients of variation for external measurements ranged from a high of 29.0 for length of hind foot in females to a low of 8.1 for length of ear in females.

Males were found to be less variable than females in most cranial measurements. The average coefficient of variation for males was 2.63 , whereas that for females was 3.79 .

\section{Geographic VARIATION}

Analysis of geographic variation was based on the examination of 59 museum specimens, mostly skins accompanied by skulls, from 22 localities in Honduras, Nicaragua, and Costa Rica. The sexes were considered together and only 


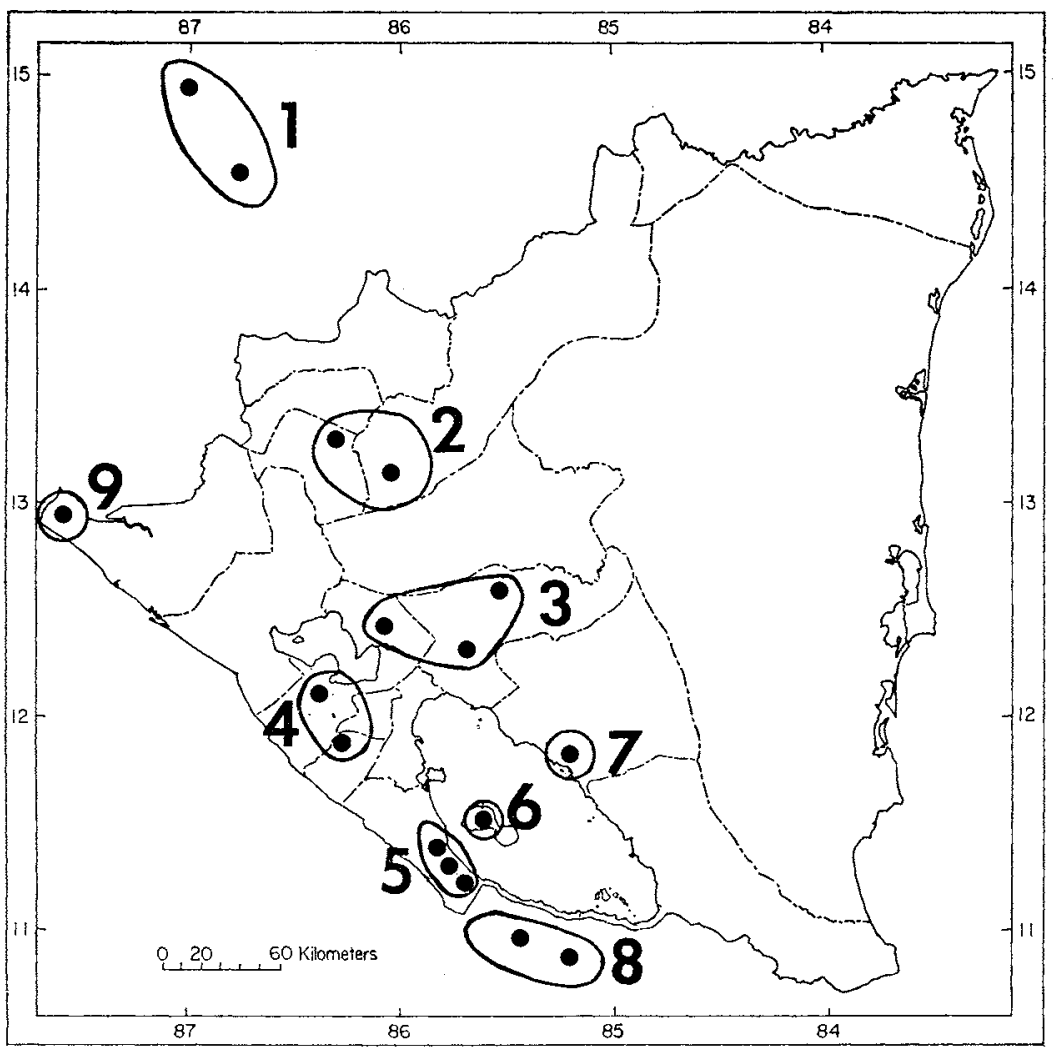

Fig. 1. - Geographic localities of samples included in the nine grouped samples of Sylvilagus floridanus. See text for localities included in each area.

individuals from age class III were included in the analysis. Most specimens examined were grouped into nine geographical samples (see Fig. 1); localities are located with reference to the nearest town : area 1-Intibuca (El Horno) and Francisco Morazán (La Flor Archaga), Honduras ; area 2-Estelf (Condega) and Jinotega (San Rafael del Norte), Nicaragua ; area 3--Boaco (Santa Rosa, Boaco) and Managua (Las Maderas), Nicaragua ; area 4-Managua (Managua) and Carazo (Diriamba), Nicaragua ; area 5-Rivas (Rivas, Finca Amayo, Sapoá), Nicaragua ; area 6-Rivas (Isla de Ometepe), Nicaragua ; area 7Chontales (Juigalpa), Nicaragua ; area 8-Guanacaste (Liberia), Costa Rica ; area 9-Chinandega (Cosigüina), Nicaragua.

Standard statistics (mean, standard deviation, and standard error) were calculated for all measurements for each area (Table 3). No evidence of clinal variation was noted for any character. Cottontails from Nicaragua averaged larger in most measurements than did those from either Honduras or Costa Rica. Those from Honduras averaged shorter in length of hind foot than did individuals from other localities. Rabbits in the Honduran sample also averaged 
smaller in greatest length of skull and length of nasals than did those in samples from Nicaragua. $S$. floridanus from Honduras exhibited a greater average zygomatic breadth than did rabbits from Nicaragua or Costa Rica.

Four different criteria (Hotelling-Lawley's Trace, Pilla's Trace, Wilk's Criterion, and Roy's maximum root criterion) were used to test for the hypothesis of no overall area effect (that is, no significant morphological difference among samples) in the MANOVA. All four tests produced $F$-values that were significant at $P<.003$; thus significant morphological differences among samples are assumed to be due to the effect of locality.

The variance-covariance matrix gave eight canonical variates among the eight characters for all nine areas. The first canonical variate expressed 54.32 per cent of the phenetic variation, the second 20.54 , the third 12.07 , and the fourth 7.50. Two dimensional plots of the first two canonical variates (including the mean and one standard deviation on each side of the mean for each area) are shown in Fig. 2. Examination of that figure reveals two major groupings of samples within the character space, one large grouping at the top consisting of samples from Nicaragua and Costa Rica and another grouping at the bottom containing only rabbits from Honduras.

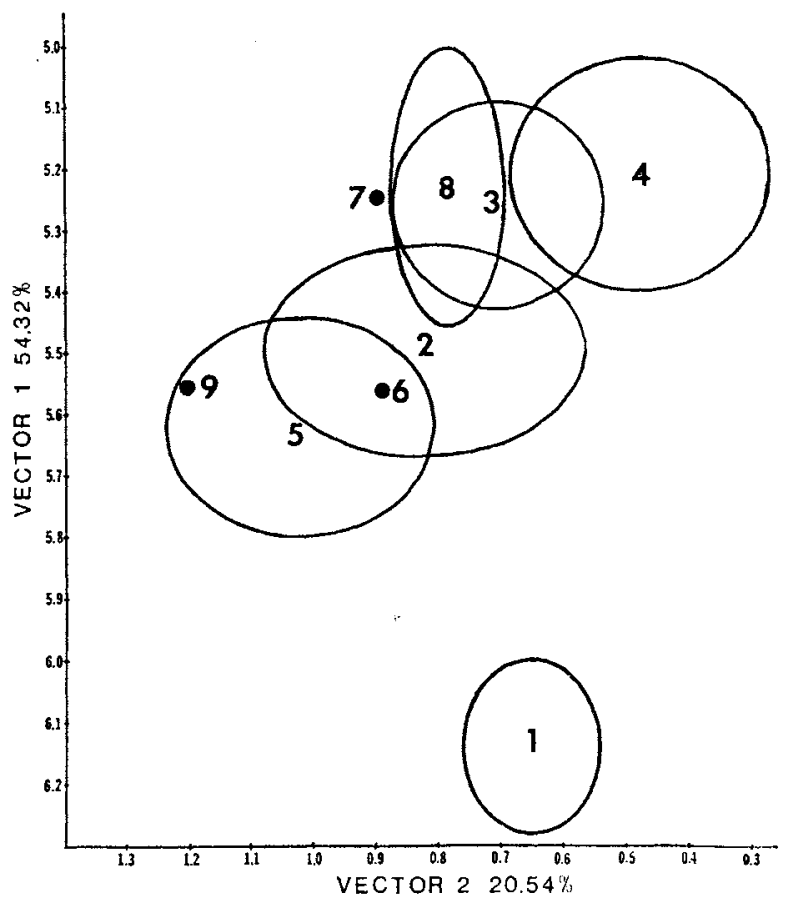

Fig. 2. - Projection of sample means ( \pm one standard deviation) for the first two canonical variates in nine samples of $S$. floridanus. Numbers represent grouped samples utilized in the study of geographic variation. Single dots represent samples with only one specimen. 
The relative contributions of each character to the first two canonical variates are given in Table 4 . The greatest degree of separation between the two groups occurs along vector I. Individuals in the upper group differ from those in the lower group in having skulls that are larger but narrower. In

TABLE 4. - Eigenvalues of selected canonical variates showing the percentage influence of each cranial character in nine samples of Sylvilagus floridanus. Eigen values shown represent the normalized vector coefficient of each character. The median is a value which reflects the approximate midpoint between the largest and smallest actual measurement of each character.

\begin{tabular}{lcrrrrrr}
\hline & & \multicolumn{2}{c}{ VECTOR I } & & \multicolumn{2}{c}{ VECTOR II } \\
Character & Median & & Eigenvalue & Per cent & & Eigenvalue & Per cent \\
\hline Greatest length of skull & 76.26 & -0.09269784 & 21.6 & & 0.1735337 & 44.35 \\
Zygomatic breadth & 34.94 & -0.31335212 & 33.4 & & 0.1295380 & 15.17 \\
Interorbital breadth & 18.97 & 0.07779589 & 4.5 & & 0.1366000 & 8.68 \\
Breadth of braincase & 25.82 & 0.14572136 & 11.5 & -0.0646280 & 5.59 \\
Length of palatal bridge & 07.36 & 0.14310542 & 3.2 & & 0.0600280 & 1.48 \\
Length of maxillary toothrow & 13.89 & 0.03810167 & 1.6 & & 0.3788000 & 17.65 \\
Breadth of mesopterygoid fossa & 08.95 & 0.00819255 & 0.2 & -0.0236400 & 0.71 \\
Length of nasals & 34.20 & 0.22892855 & 23.9 & 0.0685380 & 7.86 \\
\hline
\end{tabular}

general, the skull and nasals in samples from Nicaragua and Costa Rica averaged longer than in the Honduran sample. Zygomatic breadth averaged less in samples from Nicaragua and Costa Rica than in the one from Honduras. This is expected because greatest length of skull, length of nasals, and zygomatic breadth exert the heaviest influence on vector I. Greatest length of skull, zygomatic breadth, and maxillary toothrow exert the greatest influence on vector II.

Our analysis of geographic variation in $S$. floridanus indicates that only one race of this species, $S$. $f$. costaricensis, occurs in Nicaragua rather than S. f. hondurensis as previously believed (Goldman, 1932). When Goldman flrst described $S$. $f$. hondurensis only one specimen was available for study from Nicaragua. Likewise, when Harris (1933) described $S$. $f$. costaricensis few specimens from Nicaragua were available for comparison.

\section{Sylvilagus floridanus costaricensis Harris}

1933. - Sylvilagus floridanus costaricensis Harris, Occas. Papers Mus. Zool., Univ. Michigan, $266: 3$, June 28. Type locality, Hacienda Santa Maria, $22 \mathrm{mi}$. NE Liberia, Guanacaste, $3200 \mathrm{ft}$., Costa Rica.

Distribution. - Northwestern Costa Rica, western and central Nicaragua.

Comparisons. - From S. f. hondurensis to the north, S. f. costaricensis differs as follows : hind foot, tail, and ear longer ; tip of tail black; skull and nasals generally longer; zygomatic region narrower.

Remarks. - Sylvilagus floridanus costaricensis is restricted to the western; more arid portions of Nicaragua (Fig. 3), northward throughout the northcentral highlands where it probably intergrades with $S$. $f$. hondurensis. 


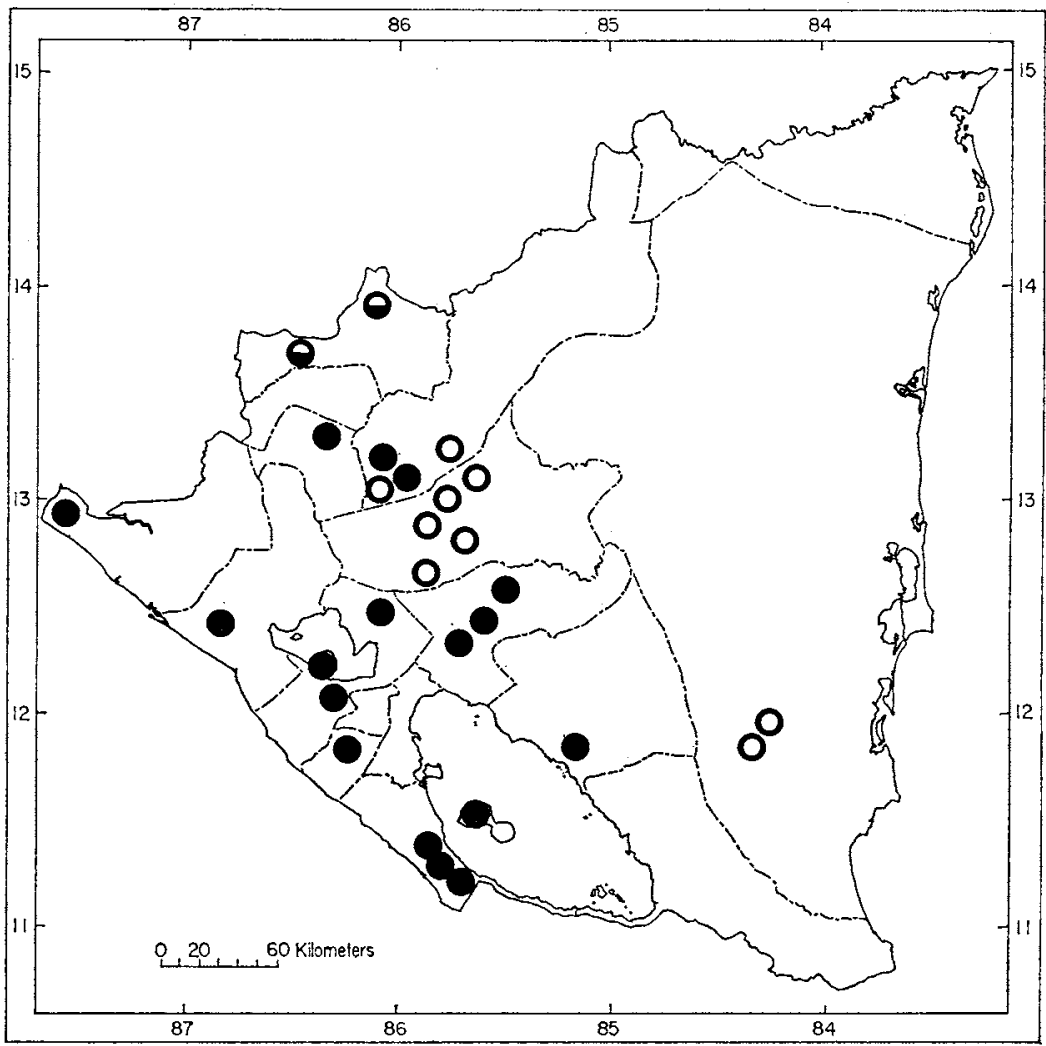

Fig. 3. - Distribution of $S$. floridanus costaricensis and $S$. brasiliensis gabbi in Nicaragua. $S$. floridanus is represented by closed circles and $S$. brasiliensis by open circles. Halfclosed circles indicate localities where both species have been taken.

The four specimens examined from Costa Rica averaged smaller than typical costaricensis, possibly because three of the four are not fully adult (age class II). There is no apparent geographic barrier to gene flow, however, between Guanacaste in Costa Rica and the adjacent Departamento de Rivas of Nicaragua. The Río Coco and several series of mountain ranges may represent substantial geographic barriers to gene flow between cottontails in Nicaragua and those in Honduras.

Testes measurements of adult males were as follows (dates of capture in parentheses) : 34 (25 March) ; 34 (21 July) ; 42 (22 July) ; 27 (11 August). Females carrying two embryos were taken at El Paraiso (20 in crown-rump length on $1 \mathrm{March}$ ) and Finca Amayo (23 on $22 \mathrm{July}$ ); females with three embryos were obtained 3 mi. SW Managua (9 on 9 March), 4 mi. SE San Rafael del Norte (58 on 12 April), and at Hato Grande (61 on 22 April). Another female taken at Hato Grande on 22 April was lactating. Nonpregnant females were collected on 21 March, 20 June, and 17 July. 
Specimens examined. - Total number 78 as follows (localities in italics are not plotted on Fig. 3) : Nicaragua. Boaco : Santa Rosa, $17 \mathrm{~km} . \mathrm{N}, 15 \mathrm{~km}$. E Boaco, 1 (KU) ; $4 \mathrm{~km}$. S, $6 \mathrm{~km}$. W Boaco, 1 (KU) ; Boaco, 1 (KU) ; Chontales, 1 (AMNH). Carazo : $3 \mathrm{~km} . \mathrm{N}, 4 \mathrm{~km}$. W Diriamba, 1 (KU). Chinandega : El Paraíso, $1 \mathrm{~km}$. N Cosigüina, 2 (KU). Chontales : Hato Grande, $13 \mathrm{~km}$. S, $8 \mathrm{~km}$. W Juigalpa, 2 (KU). Estelf : Daraili, $5 \mathrm{~km}$. N, $14 \mathrm{~km}$. E Condega, 6 (KU). Jinotega : Jinotega, 1 (USNM); San Rafael del Norte, 2 (AMNH); 4 mi. SE San Rafael del Norte, 2 (KU). León : León, 1 (AMNH). Managua : Hda. Corpus Christi, $5 \mathrm{mi}$. NE Mateape, 5 (USNM) ; $3 \mathrm{mi}$. SW Managua, 3 (KU) ; $5 \mathrm{mi}$. S Managua, 1 (KU); $10 \mathrm{mi}$. SW Managua, 1 (KU) ; $2 \mathrm{mi}$. W Las Maderas, 1 (KU). Nueva Segovia : Jalapa, 1 (AMNH) ; Ocotal, 1 (AMNH). Rivas : $4.8 \mathrm{~km}$. SE Rivas, 1 (KU) ; $7.9 \mathrm{~km}$. SE Rivas, 2 (KU) ; $12.6 \mathrm{~km}$. SE Rivas, 1 (KU) ; Hda. Amayo, $13 \mathrm{~km}$. S, $14 \mathrm{~km}$. E Rivas, 37 (21 KU, 16 USNM); $1 \mathrm{~km}$. N, $2 \mathrm{~km}$. E San Jose del Sur, Isla de Ometepe, 1 (KU); $1 \mathrm{~km}$. NW Sapoá, 1 (KU). - Costa Rica. Guanacaste : $15 \mathrm{mi}$. N Liberia, 2 (UMMZ) ; $22 \mathrm{mi}$. NE Liberia, 2 (UMMZ).

\section{SUMMARY}

The rabbits (genus Sylvilagus) of Nicaragua are herein reviewed systematically. Geographic and nongeographic variation has been assessed based on examination of 124 museum specimens constituting 28 samples of Sylvilagus floridanus from Honduras, Nicaragua, and Costa Rica, and 15 samples of $S$. brasiliensis from Nicaragua and Costa Rica. Morphological features of the skin and skull were analyzed using various univariate and multivariate statistical techniques. Analysis of geographic variation in S. floridanus indicates that rabbits of this species in Nicaragua are referable to the subspecies $S$. $f$. costaricensis. rather than to $S$. $f$. hondurensis as previously believed. Based on our assessment of geographic variation in $S$. brasiliensis, there appears to be only one subspecies in Nicaragua, S. b. gabbi.

The Museum, Texas Tech University, Lubbock, Texas 79409, U.S.A.

\section{BIBL IOGRAPHY}

Goldman, E. A., 1932. - Two new mammals from Honduras. Proc. Biol. Soc. Washington, $45: 121-124$.

Harris, W. P., 1933. - A new tree squirrel and a new cottontail rabbit from Costa Rica. Occas. Papers Mus. Zool., Univ. Michigan, $266: 1-4$.

Hershrovitz, P., 1950. - Mammals of northern Colombia. Proc. U. S. Nat. Mus., 100 : 327-375.

Nelson, E. W., 1909. - The rabbits of North America. N. Amer. Fauna, 29 : 1-314.

Power, D. M., 1970. - Geographic variation of red-winged blackbirds in central North American. Univ. Kansas Publ., Mus. Nat. Hist., 19 : 1-83.

SERVICE, J., 1972. - A users guide to the statistical analysis system. Institute of statistics, Raleigh division, North Carolina State Univ., 260 pp. 